\title{
Development of an IGF1R longevity variant mouse line using CRISPR/Cas9 genome editing
}

Yan Dou ${ }^{1}$, Martin Darvas ${ }^{2}$, Kavita Sharma ${ }^{1}$, Julie Mathieu ${ }^{1,3}$, John Morton ${ }^{1}$, Heidi Tan ${ }^{1}$, Carolina Soto-Palma ${ }^{4}$, Luise A Angelini ${ }^{4}$, Sara J McGowan $^{4}$, Laura J Niedernhofer ${ }^{4}$, Yousin Suh ${ }^{5}$, Paul D Robbins ${ }^{4}$, Nir Barzilai ${ }^{6}$ and Warren C Ladiges ${ }^{*}$

${ }^{1}$ Department of Comparative Medicine, School of Medicine, University of Washington, Seattle, WA, USA

${ }^{2}$ Department of Pathology, School of Medicine, University of Washington, Seattle, WA, USA

${ }^{3}$ Institute for Stem Cell and Regenerative Medicine, School of Medicine, University of Washington, Seattle, WA, USA

${ }_{4}^{4}$ Institute on the Biology of Aging and Metabolism, Department of Biochemistry, Molecular Biology and Biophysics, University of Minnesota, Minneapolis, MN, USA

${ }^{5}$ Department of Genetics and Development, Columbia University, New York, NY, USA

${ }^{6}$ Department of Medicine, Department of Genetics, Institute for Aging Research, Albert Einstein College of Medicine, New York, NY, USA

\begin{abstract}
An insulin-like growth factor-1 receptor (IGF1R) variant in exon 6 (Arg-407-His) in Ashkenazi Jewish centenarians was previously found to be associated with reduced IGF1R activity. To further study this longevity associated IGF1R variant, we generated a novel mouse line carrying the R407H variant in exon 6 of the Igf1r gene by employing CRISPR/Cas9 genome editing technology. Here, we show that the Igf1r gene can be edited in mouse embryos by zygotic electroporation of Cas 9 protein and a single-guide RNAs together with a single stranded oligonucleotide donor containing the desired key nucleotide changes at the Igflr locus. Sequence analysis of F0 and F1 mice following targeted editing demonstrated the robustness of this approach in mice using CRISPR/Cas 9 directed homologous recombination (HDR). Western blot analysis indicates that mice heterozygous for the variant have a significant decrease in IGF1R phosphorylation in various tissues, including skeletal muscle, compared to wildtype. In addition, depletion of IGF1R signaling specifically in skeletal muscle of progeroid $E r c c 1^{-/ \Delta}$ mice resulted in extended health span and median lifespan providing the rationale for long term lifespan studies in $\operatorname{Ig} f 1 \mathrm{r}$ hR $407 \mathrm{H}$ variant mice. This mouse line will be a valuable genetic tool to help determine the impact of IGF1R signaling on aging and longevity. The CRISPR editing approach represents a prototype for generating additional longevity associated gene variant mouse lines to study relevance to human exceptional longevity.
\end{abstract}

\section{Introduction}

Loss of function in genes encoding components of insulin/insulinlike growth factor-1 (IGF1) signaling have been shown to extend lifespan in yeast, worms, flies, and mice [1]. While insulin mainly provides short-term effects that regulate metabolic pathways, IGF1 promotes chronic effects that determine cell fates, such as growth or differentiation of target cells, cell survival and maintenance of cell function [2]. The relevance of this regulatory network for exceptional longevity in humans remains unproven. Studies have reported on levels of IGF1 and related molecules in centenarians, but these lacked adequate controls. This shortcoming was overcome by establishing a cohort of Ashkenazi Jewish decent with extended longevity and their offspring, and age- and sex-matched controls without a family history of unusual longevity [3]. Using this group of individuals, variants in the $I G F 1 R$ were found to be enriched in centenarians compared to controls. Immortalized lymphocytes from carriers of two nonsynonymous variants, A37T and $\mathrm{R} 407 \mathrm{H}$, showed significant reduction in IGF1R and its phosphorylation levels [3]. In a follow up study, engineered to express the two variants in $I g f 1 r^{-1}$ mouse embryonic fibroblasts (MEFs) also showed reduced phosphorylation of IGF1R [4].

These observations provided the rationale to further investigate the functional significance of the $I G F 1 R$ variants in a genetically engineered mouse model and determine the impact on longevity. CRISPR/Cas9 genome editing is an established technology for generating genetic alterations in mice. However, it has not been applied to the development of mice expressing human longevity gene variants. In this report, we describe a CRISPR/Cas9 protocol to generate a mouse line with the human $I G F 1 R$ variant $\mathrm{R} 407 \mathrm{H}$ in exon 6 , designated as $I G F 1 R^{\mathrm{hR} 407 \mathrm{H}}$ mice. Our data demonstrate that $I G F 1 \mathrm{R}^{\mathrm{hR} 407 \mathrm{H}}$ mice have reduced IGF1R activity in multiple organs. In addition, a kinase-dead mutation in IgfIr extended the health span and median lifespan of progeroid $\mathrm{Erccl}^{-/ \Delta}$ mice, consistent with lower IGF1R signaling in specific tissues contributing to the longevity and health of centenarians, which provides the rationale for lifespan studies in $I G F 1 R^{\mathrm{hR} 407 \mathrm{H}}$ mice. This approach can also be used to examine the role of additional rare variants identified in centenarians (Zhengdong, et al. accepted for publication in Nature Metabolism).

\section{Methods}

\section{Animals}

Mice were housed in an SPF facility at the University of Washington under a 12-hour light and 12-hour dark cycle with room temperature

${ }^{\star}$ Correspondence to: Warren Ladiges, Department of Comparative Medicine, School of Medicine, University of Washington, Seattle, WA, USA, E-mail: wladiges@uw.edu

Key words: human longevity, centenarians, IGF1R variants, CRISPR/Cas9 gene editing, Igflr variant mouse model

Received: September 28, 2020; Accepted: October 05, 2020; Published: October 08,2020 
of $25^{\circ} \mathrm{C} \pm 4$. Reverse osmosis water and irradiated food (Picolab Rodent Diet 20, 5053) were supplied. All studies were approved by the University of Washington IACUC. Mice were also kept in an SPF facility at the University of Minnesota under similar conditions. Mutant Igfr1 (FVB-Tg (Ckm-IGF1R $\left.\left.{ }^{\star} \mathrm{K} 1003 \mathrm{R}\right) 1 \mathrm{Dlr} / \mathrm{J}\right)$ mice $\left(I g f 1 r^{t g}\right)$ were obtained from the Jackson Laboratory (Stock \#016618). The animals harbor a K1003R mutation that inactivates the ATP-binding site in the enzyme, preventing autophosphorylation and phosphorylation of substrates ERK and AKT. Expression is driven by the creatine kinase muscle-specific promoter $(\mathrm{Ckmm})$ and there are numerous copies of the transgene leading to strong expression of the mutant receptor in skeletal muscle and much lower expression in the heart [5]. These mice were crossed with inbred FVB Ercc1 ${ }^{+/ \Delta}$ mice. Then $I g f 1 r^{\text {tt; }} \mathrm{Erccl}^{+/ \Delta}$ progeny further crossed with inbred C57BL/ $6 \mathrm{Erccl}^{+/-}$mice to generate F1 Igfl $r^{t g} ;$ Ercc $^{-/ \Delta}$ experimental mice. Breeding, health span and lifespan studies were approved by the UMN IACUC.

\section{CRISPR construct design}

The human IGF1R gene encodes a mature protein with a length of 1367 amino acids (aa) and the mouse Igflr gene encodes mature protein with a length of $1369 \mathrm{aa}$. Human and mouse IGF1R mature proteins result from cleavage of a 30 -aa N-terminal signal peptide (Supplementary Figure 1) and amino acid numbering for IGFR is based on the mature protein [3]. A single-guide RNA (sgRNA) for Streptococcus pyogenes Cas9 (SpCas9) was used to mediate editing of the murine Igfir gene. We submitted the nucleotide sequence containing Igflr exon 6 to the Broad Institute Genetic Perturbation Platform (GPP) web portal [6-8]. We identified the sgRNA ${ }^{I g f t r}$ sequence ACCUGACGGUCAGGUUCCGG as a candidate for editing the part of exon 6 in the Igflr gene that needs to be modified for the generation of $I G F 1 \mathrm{R}^{\mathrm{hR} 407 \mathrm{H}}$ mice at the mouse 408 residue position. Because of the different lengths of mouse and human IGF1R, the mouse arginine at position 408 corresponds to the human arginine at position 407 (Supplementary Figure 1). We used a 123-bp synthetic single-stranded DNA oligo donor (IDT, San Jose CA) that was based on the wild-type Igflr sequence and contained two mutations that would result in destruction of the PAM sequence for sgRNA ${ }^{\operatorname{Igfir}}$ (TGG > TGA, synonymous mutation), and an amino-acid change from R to $\mathrm{H}$ (c.CGG > CAC, p.R408H).

\section{Generation and sequencing of pups}

C57BL/6 female mice, 3-4 weeks of age, were super-ovulated by intraperitoneal injection of $5 \mathrm{IU}$ of PMSG, followed by intraperitoneal injection of 5 IU hCG 48 hours later. Super-ovulated females were mated with adult C57BL/6 stud males and euthanized the next morning. Zygotes were harvested from the oviducts in M2 solution. Ribonucleoprotein (RNP) complex was prepared with $2 \mu \mathrm{M}$ SpCas9 mRNA (Sigma) and $2 \mu \mathrm{M}$ of sgRNA against Igflr exon 6 (Synthego, Menlo Park CA) and incubated for $15 \mathrm{~min}$ at room temperature. Two batches of 37 embryos were electroporated with the RNP complex and $10 \mu \mathrm{M}$ of single-stranded DNA oligo donor (IDT) in electroporation buffer [9] using a Bio-Rad (Hercules CA) electroporator (GenePulser Xcell: $30 \mathrm{~V}, 10 \mathrm{~ms}, 4$ pulses) and cultured in KSOM media for $24 \mathrm{hrs}$. Twenty-nine 2-cell embryos were surgically transferred into the oviduct of two pseudopregnant mice $(14+15)$. Three pups were born, and DNA was isolated three weeks later from tail tips using DNeasy extraction kit (Qiagen, Germantown MD). Nested PCR was performed around Igfir sgRNA target site (oligo F: 5' CCAGTAAGCAAGCAAACCCCT 3' and oligo R: 5' CACGGATGCCAAAGACTGTTA 3'). The PCR products were purified using EXO-SAP enzyme (ThermoFisher Scientific, Waltham MA) and sent for Sanger sequencing analysis (through Genewiz, Seattle WA) to check the genotyping of the mice.

\section{Genotyping}

Briefly, tail biopsies from 8 to 10-day old pups were digested overnight at $37^{\circ} \mathrm{C}$ in tissue lysis buffer containing $10 \mathrm{mM}$ Tris, 5 $\mathrm{mM}$ EDTA, $100 \mathrm{mM} \mathrm{NaCl}, 1 \%$ SDS and $50 \mu \mathrm{g} / \mathrm{ml}$ proteinase $\mathrm{K}$. The CRISPR nuclease targeted regions were PCR amplified with high-fidelity polymerase (Platinum Taq DNA Polymerase High Fidelity, Invitrogen). To detect gene edition, locus specific primers pair (oligo F: 5' CAAGCACATTGTCCATACCTG 3' and oligo R: 5'CACGGATGCCAAAGACTGTTA 3') were used. PCR conditions were as follows: 35 cycles at $95^{\circ} \mathrm{C}$ for $30 \mathrm{~s}, 57^{\circ} \mathrm{C}$ for $30 \mathrm{~s}$, and $72^{\circ} \mathrm{C}$ for $30 \mathrm{~s}$. PCR products from wildtype and transgenic mice were subjected to TFiI restriction endonuclease to confirm the presence of the desired mutation. Restriction digestion was carried out at $65^{\circ} \mathrm{C}$ for $30 \mathrm{~min}$, followed by gel electrophoresis for product size visualization on a $2 \%$ agarose gel. UMN mice were genotyped by TransnetYX (Cordova, TN).

\section{Healthspan and lifespan analysis of progeria mice}

Igflr ${ }^{\text {tg; }} \mathrm{Erccl}^{-/ \Delta}$ and $\mathrm{Erccl}^{-/ \Delta}$ mice were monitored for changes in health status twice per week beginning at weaning. The animals were monitored for symptoms listed in Table 1 and scored. Lifespan was measured by scoring deaths or terminal condition requiring euthanasia.

\section{Enzyme-linked immunosorbent assay (ELISA)}

An ELISA kit was used to determine the level of IGF1 in the serum of mice (R\&D System, UK) according to the manufacturer's protocol. Briefly, serum samples from 6-month-old female mice were added into each well of a pre-coated ELISA plate and incubated at room temperature for $120 \mathrm{~min}$. After that, wells were washed 5 times and an HRP-conjugated antibody was added, followed by incubation for $120 \mathrm{~min}$. Next, wells were washed 5 times, substrate solution was added and incubated for $30 \mathrm{~min}$. Then, stop solution was added and the absorbance at $450 \mathrm{~nm}$ was determined using a microplate reader (Synergy H1, BioTek, Winooski, VT, USA).

\section{Immunoblots}

Heart, kidney, pancreas, and skeletal muscle were isolated from 6-month old mice and snap frozen at $-80{ }^{\circ} \mathrm{C}$. Protein extraction was carried out using T-PER ${ }^{\mathrm{mix}}$ Tissue Protein Extraction Reagent (ThermoFisher Scientific, Waltham MA) with Halt ${ }^{\mathrm{su}}$ Protease Inhibitor Cocktail (ThermoFisher Scientific, Waltham MA) and Halt ${ }^{\mathrm{tw}}$ Phosphatase Inhibitor Cocktail (ThermoFisher Scientific, Waltham MA). Protein concentration of each sample was determined by BCA Protein Assay Reagent (Pierce, Rockford IL). Standard electrophoresis was performed. Blocking and antibodies were prepared in filtered 5\% BSA. The blots were developed using Clarity Max Western ECL Substrate (Bio-Rad, Hercules CA) and were scanned using Azure Imager C300 (Azure Biosystems, Dublin CA). The densitometric analysis was conducted to quantify the Western blot immunoreactivity using Image J. The primary antibodies used were IGF-I Receptor $\beta$ (1:1000, \#9750S), Phospho-IGF-I Receptor $\beta$ (1:1000, \#3918S), Akt (pan) (1:1000, \#4691S), Phospho-Akt (1:2000, \#4060S), and Actin (pan) (1:1000, \#4968S). All primary antibodies were obtained from Cell Signaling Technology (Danvers MA). The secondary antibody used was goat anti-rabbit IgG-HRP (1:4000; Santa Cruz Biotechnology, Santa Cruz CA).

\section{Statistical analysis}

All the data are presented as mean \pm standard error of mean (SEM) or \pm standard deviation (SD). Statistical comparisons were performed with the independent $t$-test. The criterion for statistical significance was 
Table 1. Symptoms and scoring severity to calculate an aging score, which is a measure of health span

\begin{tabular}{|c|c|c|c|}
\hline Symptoms & Test & Score & Description \\
\hline \multirow{3}{*}{ Kyphosis } & \multirow{3}{*}{$\begin{array}{l}\text { Inspect the mouse for curvature of the spine or hunched } \\
\text { posture. Run your fingers down both sides of the spine to detect } \\
\text { abnormalities. }\end{array}$} & 0 & Absent \\
\hline & & 0.5 & Mild curvature \\
\hline & & 1 & Clear evidence of hunched posture \\
\hline \multirow{3}{*}{ Ataxia } & \multirow{3}{*}{ Observe gait and stance on a smooth surface } & 0 & Absent \\
\hline & & 0.5 & Occasional non-toe spread in stance and gait \\
\hline & & 1 & Ataxia postures present (no toe spread, foot dragging) \\
\hline \multirow{3}{*}{ Gait disorders } & \multirow{3}{*}{$\begin{array}{l}\text { Observe the freely moving animal to detect abnormalities such as } \\
\text { hopping, wobbling, circling, wide stance and weakness. }\end{array}$} & 0 & No abnormality \\
\hline & & 0.5 & Abnormal gait but animal can still walk \\
\hline & & 1 & Marked abnormality, impairs ability to move \\
\hline \multirow{5}{*}{ Dystonia } & \multirow{5}{*}{ Hold Mouse by tail to for 20 secs and observe hind limb splay } & 0 & No motor abnormalities \\
\hline & & 0.25 & One or both legs are drawn in within 20 secs \\
\hline & & 0.5 & One or both legs are drawn in within 10 secs \\
\hline & & 0.75 & One leg Is drawn in immediately \\
\hline & & 1 & Both legs are drawn in immediately \\
\hline \multirow{3}{*}{ Tremor } & \multirow{3}{*}{$\begin{array}{l}\text { Observe the freely moving animal to detect tremor, both at rest } \\
\text { and when the animal is trying to climb up an incline. }\end{array}$} & 0 & No tremor \\
\hline & & 0.5 & Slight tremor \\
\hline & & 1 & Marked tremor \\
\hline \multirow{3}{*}{ Body condition } & \multirow{3}{*}{$\begin{array}{l}\text { Place mouse on flat surface, hold tail base and manually assess the } \\
\text { flesh/fat that covers the sacroiliac region (back and pubic bones). }\end{array}$} & 0 & Bones palpable. not prominent \\
\hline & & 0.5 & Bones prominent or barely felt \\
\hline & & 1 & Bones very prominent or not felt due to obesity \\
\hline
\end{tabular}

chosen to be $\mathrm{p}<0.05$. For animal studies, mice harboring a mutation in Igflr or not were compared in a progeroid $\mathrm{Erccl}^{-1 \Delta}$ background using an unpaired Student's t-test.

\section{Results and discussion} editing

The generation of IGF1R ${ }^{\mathrm{hR} 407 \mathrm{H}}$ mice using CRIPSR/Cas9 gene

We generated a mouse line that replicates the human IGF1R R407H longevity-related variant observed in Ashkenazi Jewish centenarians using CRIPSR/Cas9 technology to generate an R408H substitution in the mouse Igfl $r$ gene. The first step was electroporation of the RNP consisting of the sgRNA ${ }^{I g f l r}$ and SpCas9 mRNA with a 123-bp single stranded oligonucleotide donor template (ssODN) into one-cell mouse zygotes. We designed the ssODN having three base mismatches to the genomic sequence to introduce the CGG > CAC substitution that changes the Arg at mouse position 408 to His by HDR, and to direct the synonymous mutation (TGG $>$ TGA) at the Protospacer Adjacent Motif (PAM) sequence to destroy the PAM with introduction of a Tfil restriction site at the same time for later mouse genotyping (Figure 1A). After electroporation and overnight culture, the two-cell embryos were surgically transferred into the oviduct of pseudopregnant mice. The founder pups were genotyped and sequenced for the three nucleotide substitutions (Figure 1B). The line was designated as $\mathrm{h}$ (human) $\mathrm{R} 407 \mathrm{H}$ (IGF1R $\mathrm{R}^{\mathrm{hR} 407 \mathrm{H}}$ ) to show that at mouse position 408 , the human $\mathrm{R} 407 \mathrm{H}$ variant was correctly represented (Supplementary Figure 1).

Three pups were born and screened for the presence of the hR407H mutation using PCR amplification and DNA sequencing. Two of the pups had the hR407H mutation present with the desired mutation at the PAM region in their genome while the third pup had only random insertions/deletions. In all 3 mice, Sanger sequencing showed that additional sequences were present in the DNA extracted from the tails, revealing a high degree of mosaicism (Figure 1D), which has been commonly reported in CRISPR edited mice [10]. We bred the two F0 founder mice with C57BL/6 wildtype mice and assessed the germline transmission of the IGF1R hR407H allele. We genotyped the F0 founders and F1 mice using PCR and restriction endonuclease digestion at the TfiI sites to confirm the presence of the desired mutation. Since the amplified region from wildtype mice had only one TfiI site whereas heterozygous IGF1R hR407H mice had two, the product sizes of wildtype mice samples were 61 base pairs (bps) and 544 bps, whereas the product sizes from IGF1R hR407H mice were $61 \mathrm{bps}, 225 \mathrm{bps}, 319$ bps, and 544 bps after the restriction digestion (Figure 1C). At the end, 9 out of 13 F1 pups obtained from both founders were heterozygous for the hR407H mutation, with further sequencing data demonstrating the desired mutations with no off-target editing, indicating high efficiency and accuracy of our design. (Figure 1C, D).

\section{Expression levels of IGF1R were altered in female mice with the IGF1 $\mathrm{R}^{\mathrm{hR} 407 \mathrm{H}}$ genotype}

After successfully generating the $\mathrm{F} 1$ heterozygous IGF1R $\mathrm{R}^{\mathrm{h} 407 \mathrm{H}}$ genotype, we searched for alterations in IGF1R function. Since a previous study of $\mathrm{R} 407 \mathrm{H}$ centenarian carriers showed a more robust impaired-IGF1 signaling phenotype in females but not in males [3], we first measured serum IGF1 levels in 6-month-old wildtype (WT) and $\mathrm{IGF}^{\mathrm{h}} \mathrm{R}^{\mathrm{h} 407 \mathrm{H}}$ female mice. We found there was no difference in the IGF1 serum concentration between WT and mutant female mice (Figure $2 \mathrm{~A}$ ), in contrast with the results seen in female centenarian offspring whose serum IGF1 concentrations were significantly higher than those of controls. To further investigate changes in IGF1R signaling in mutant female mice, we analyzed the phosphorylation of IGF1R and AKT in heart, kidney, pancreas, and skeletal muscle. We found a significant decrease In IGF1R phosphorylation levels in mutant mice compared to WT mice in heart, kidney, and skeletal muscle, but not in the pancreas (Figure 2B-E). Interestingly, there was a two-fold increase in the IGF1R expression in IGF1 $\mathrm{R}^{\mathrm{hR} 407 \mathrm{H}}$ mice in all the tissues, with a significant increase in heart, kidney, and skeletal muscle (Figure 2B-E). However, no significant decrease in the phosphorylation level of AKT was observed in any of the tissues, although there was a downward trend observed in the kidney (Figure 2B-E).

The decreased IGF1 signaling level in IGF1 $\mathrm{R}^{\mathrm{hR} 407 \mathrm{H}}$ female mice helps to verify the integrity of the mouse line and relevance as a model for the human longevity-related mutation. However, our IGF1R ${ }^{\text {hR407H }}$ mice show a discrepancy from the human centenarian offspring carriers in terms of no significant increase in serum IGF1 level and no significant 
A

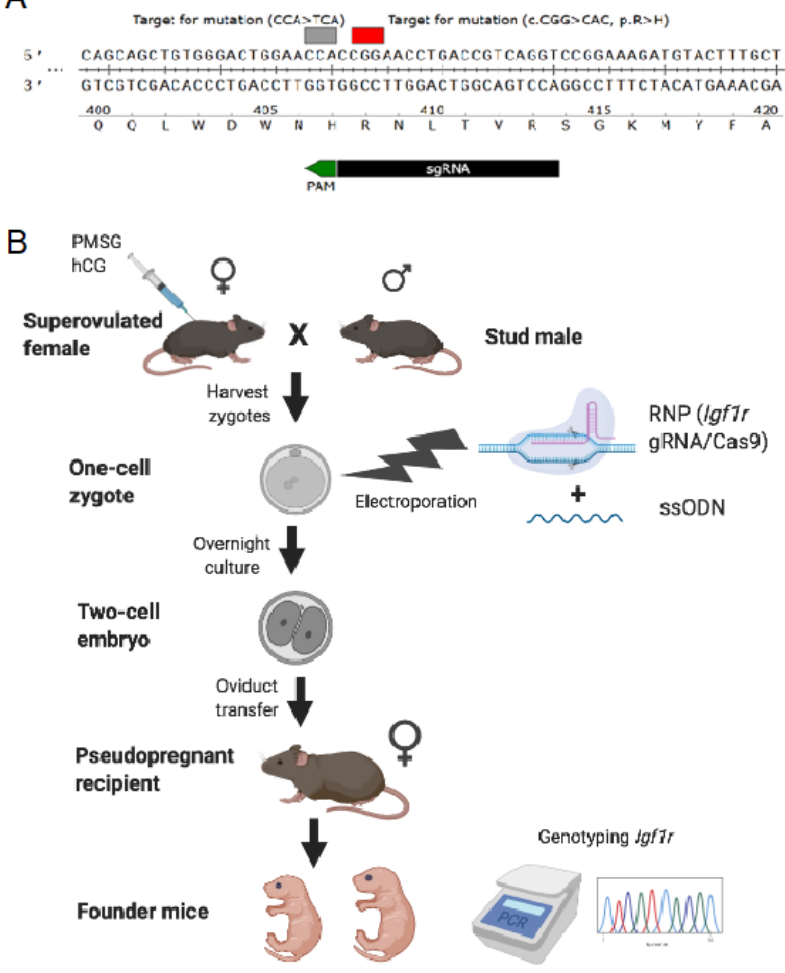

C
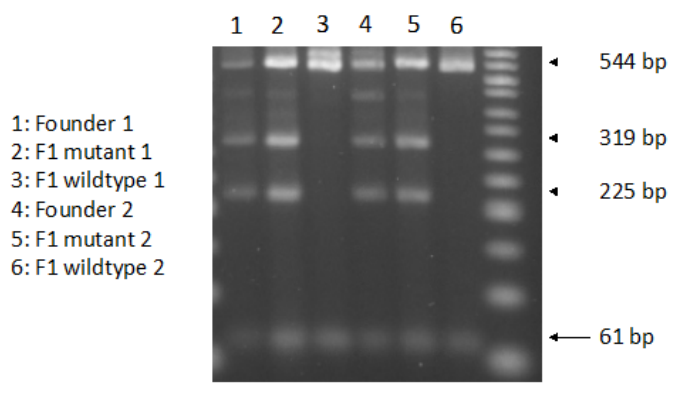

D Sequencing founder and F1 heterozygous mice

$\downarrow$

Wild type

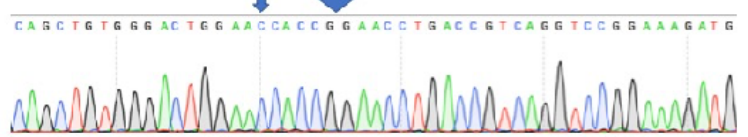

Mosaic
founder

founder
$\# 1$

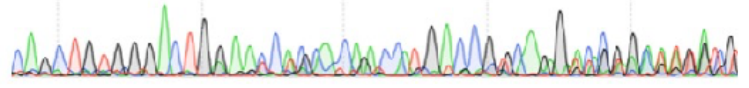

Mosaic

founder

$\# 2$

Heterozygous

F1 (244)

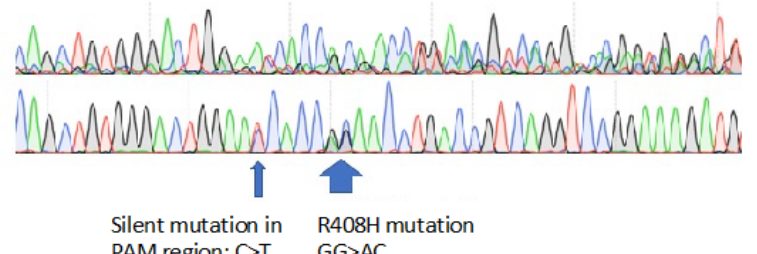

PAM region: $O T \quad G G>A C$

Figure 1. IGF1R $\mathrm{R}^{\mathrm{hR} 407 \mathrm{H}}$ mice were generated using CRIPSR/Cas9 editing. A. Schematic of the Igfl $r$ targeting strategy. The sgRNA ${ }^{\text {Igf }}$ is indicated in black and its PAM sequence in green. The mutation introduced in amino acid $408(\mathrm{R}>\mathrm{H}$ : $\mathrm{CGG}>\mathrm{CAC})$ and the silent mutation in the PAM sequence (CCA $>$ TCA) are indicated in red and grey, respectively. B. Experimental workflow. Female mice were super-ovulated using PMSG and hCG and housed with stud males for breeding. One-cell zygote embryos were collected and electroporated with sgRNA ${ }^{\text {Igf } I} / \mathrm{Cas} 9 \mathrm{RNP}$ complex and ssODN. The next day, embryos were transferred to pseudo-pregnant recipient to generate edited mice. The resulting pups were genotyped by PCR and Sanger sequencing analysis. C. Electrophoresis gel showing the PCR fragments pattern from two founders and their wildtype and heterozygous F1 IGF1R ${ }^{\mathrm{hR} 407 \mathrm{H}}$ mice after digestion at the Tfil restriction sites. D. Sanger sequencing analysis of exon 6 of Igflr in F0 founder mice and one of the heterozygous F1 mice (pup 244)

decrease in IGF1R level in the tissues [3]. In fact, the significant increase in IGF1R expression in multiple organs suggests a potential positive feedback mechanism between the IGF1R $\mathrm{R}^{\mathrm{hR} 407 \mathrm{H}}$ variant and expression of IGF1R independent of IGF1 levels in mutant mice. This might indicate differences between human and mice in terms of how the IGF1R $\mathrm{R}^{\mathrm{h} 407 \mathrm{H}}$ variant induces a feedback mechanism to compensate for the reduction of IGF1 signaling level. The failure to observe any difference in the downstream signaling of AKT in IGF1 $\mathrm{R}^{\mathrm{hR} 407 \mathrm{H}}$ mice could be related to the young age of the mice. More significant changes may be seen when older animals become available for study. In addition, actual studies on signaling in response to an IGF1 bolus would help to establish physiological proof that IGF1R signaling is downregulated in IGF1R ${ }^{\mathrm{hR} 407 \mathrm{H}}$ mice.

\section{Overexpression of kinase-dead IGF1R in skeletal muscle extends the healthspan and median lifespan of progeroid Ercc1 $^{-/ \Delta}$ mice}

Since we found a significant decrease in IGF1R phosphorylation level in skeletal muscle of IGF1R $\mathrm{R}^{\mathrm{hR} 407 \mathrm{H}}$ mice, we wanted to determine if reduced IGF1R signaling specifically in skeletal muscle could promote healthspan and lifespan. To facilitate these experiments, the $\mathrm{Erccl}^{-1 / \Delta}$, which model a human progeroid syndrome and have an accelerated aging phenotype [11] was utilized. The $\operatorname{Erccl}^{-1 \Delta}$ mice were crossed with mice expressing in skeletal muscle an IGF1R with a K1003R mutation that inactivates the ATP-binding site in the enzyme, preventing autophosphorylation and phosphorylation of substrates in skeletal muscle upon ligand binding.

Immunoblot analysis of protein isolates prepared from quadricep muscle indicates strong overexpression of IGF1R in skeletal muscle of the transgenic mice (Figure 3A-B). Phosphorylation of IGF1R is already low in $\operatorname{Erccl}^{-1 \Delta}$ mice consistent with suppression of the IGF1 axis, previously reported in $\mathrm{Erccl}^{-1}$ mouse tissues. AKT expression was unchanged in skeletal muscle of the transgenic mice (Figure 3C-D). However, phosphorylation was increased, albeit inconsistently, in the skeletal muscle of $\operatorname{Igf1R^{\mathrm {Tg}}}$; $\operatorname{Erccl}^{-/ \Delta}$ mice compared to $\operatorname{Erccl}^{-/ \Delta}$, as was previously reported for $I g f 1 R^{\mathrm{Tg}}$ mice.

A cohort of $\operatorname{Igfl} 1 \mathrm{r}^{\mathrm{tg}}$; $\mathrm{Erccl}^{-1 \Delta}$ mice were bred and their health monitored across their lifespan and compared to $\mathrm{Erccl}^{-1 /}$ mice. As previously reported [5], mice harboring the Igf1R mutant transgene had significantly reduced body weight compared to littermate controls (Supplemental Figure 2). The Igflr ${ }^{T g} ; \operatorname{Erccl}^{-/ \Delta}$ mice showed a significant delay in the onset of age-related symptoms characteristic of progeroid $\operatorname{Erccl}^{-/ \Delta}$ mice (Figure 4A). Igf1rts; $\operatorname{Erccl}^{-/ \Delta}$ mice also showed a dramatic extension of median lifespan compared to progeroid $\mathrm{Erccl}^{-1 / \Delta}$ mice (Figure 4B), consistent with an extension of healthy aging. As a result, depletion of IGF1R signaling specifically in skeletal muscle can increase the health span and medium lifespan of progeroid mice, providing the rationale for conducting lifespan and healthspan studies in IGF1R $\mathrm{R}^{\mathrm{hR} 407 \mathrm{H}}$ mice. 
A

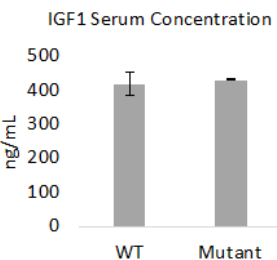

B

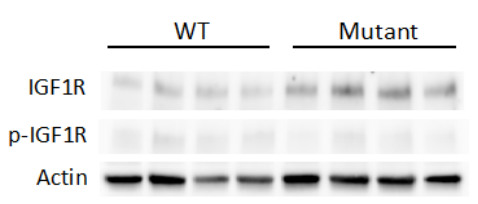

C

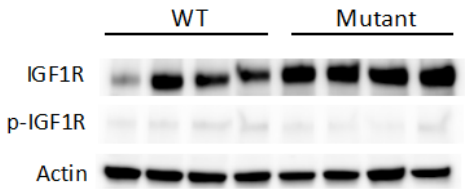

$\mathrm{D}$

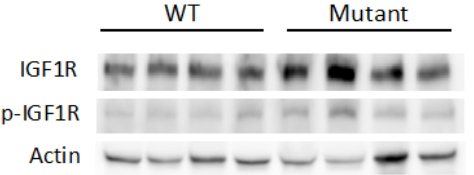

$E$

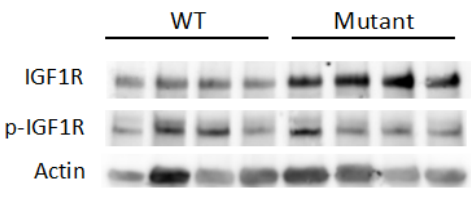

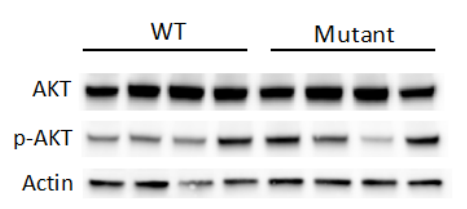
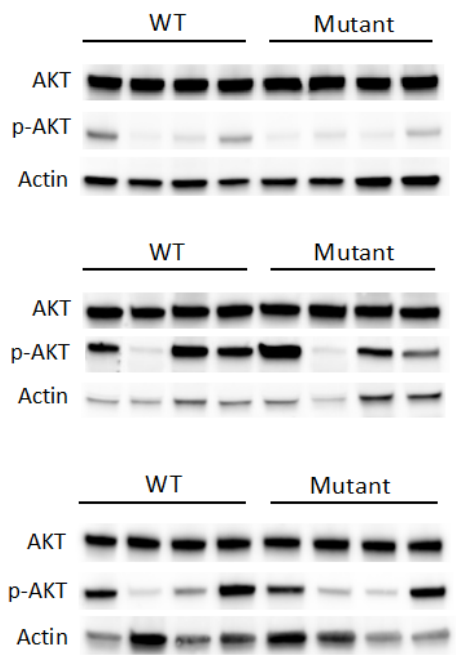

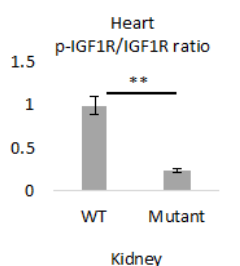

Kidney
1.5 p-IGF1R/IGF1R ratio
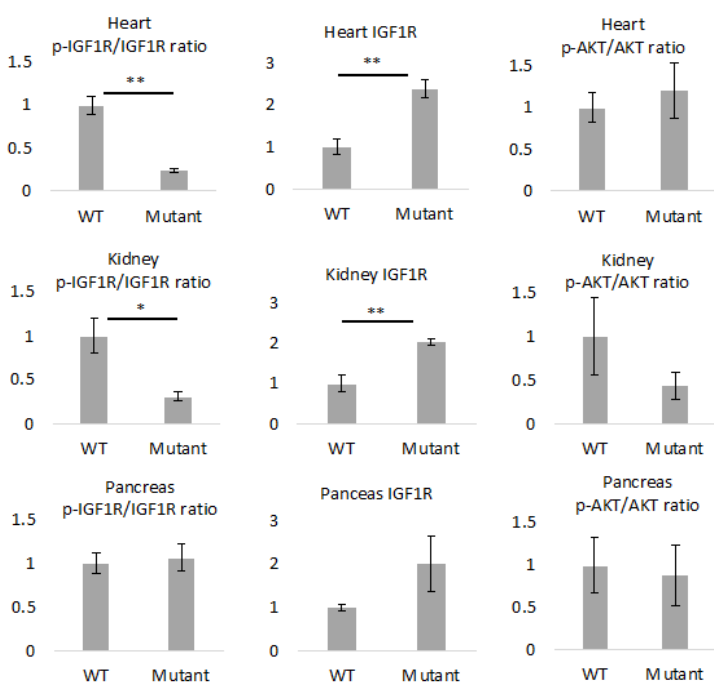

Kidney IGF1R

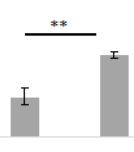

$$
\begin{array}{r}
1.5 \\
1 \\
0.5 \\
0
\end{array}
$$

$$
\text { Kidney }
$$

Kidney
-AKT/AKT ratio

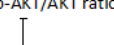

WT Mutant

Panceas IGF1R
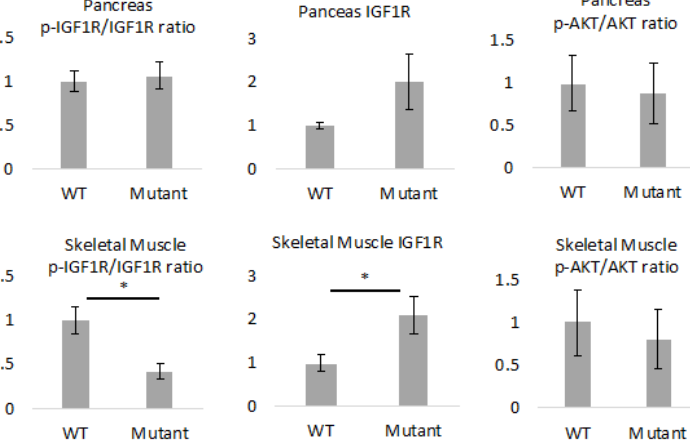

\section{Skeletalmuscle} p-AKT/AKT ratio
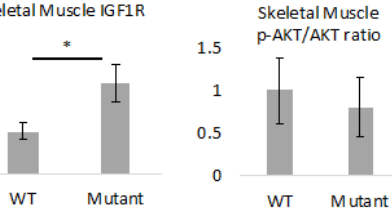

Figure 2. Female mice with the IGF1R $\mathrm{R}^{\mathrm{hR} 407 \mathrm{H}}$ genotype showed alterations in IGF1R expression levels. A. Serum IGF1 levels in female WT and IGF1R $\mathrm{R}^{\mathrm{hR} 407 \mathrm{H}}$ (mutant) mice at 6 months of age $(\mathrm{n}=4-5)$. B-E. Western blotting was performed to measure the phosphorylation levels of IGF1R, AKT and the total IGF1R expression in heart (B), kidney (C), pancreas (D), and skeletal muscle (E) tissues ( $\mathrm{n}=4$ mice per genotype). The phosphorylated to total protein ratios and IGF1R total protein expression, as determined by densitometry, are shown on the right (B-E). IGF1R expression levels were normalized to each individual actin. All data represent the mean $\pm \mathrm{SEM}$. ${ }^{*} \mathrm{p}<0.05,{ }^{* *} \mathrm{p}<0.01$, statistically significant difference between WT and IGF1R ${ }^{\mathrm{hR} 407 \mathrm{H}}$ mice

A

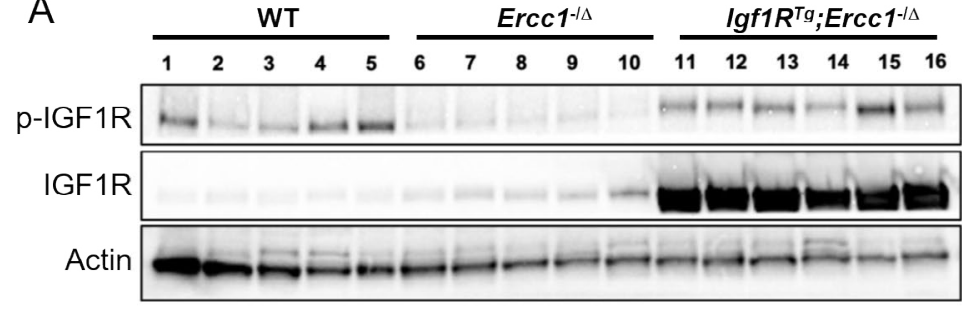

C

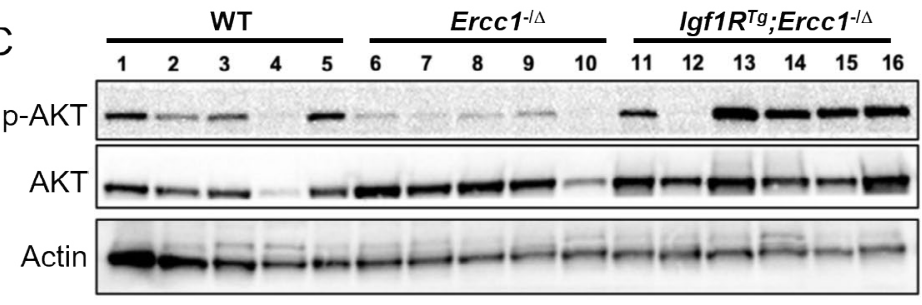

$\mathrm{B}$

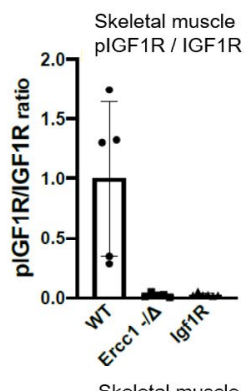

D

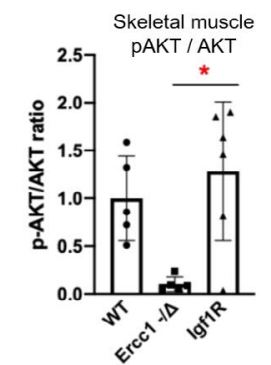

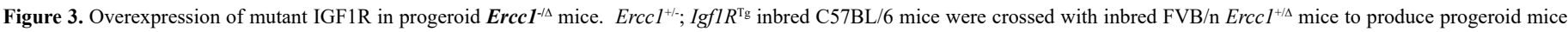
harboring the IgflR transgene driving over expression of mutant IGF1R in differentiated myocytes from the creatinine-kinase muscle-specific promoter. A. Immunoblot detection of IGF1R expression and the auto-phosphorylated receptor in skeletal muscle of the mutant animals compared to controls. B. Quantitation of the immunoblot data using ImageJ. Values are standardized using Actin as a loading control and reported is the ratio of the phosphorylated receptor to non-phosphorylated. C. Immunoblot detection of AKT and phosphorylated AKT in skeletal muscle of the same animals. D. Quantitation of the immunoblot data using ImageJ. Values are standardized using Actin as a loading control and reported is the ratio of the phosphorylated receptor to non-phosphorylated. Shown are the mean + - S.D. Paired t-test, ${ }^{*} \mathrm{p}<0.05$ 
A

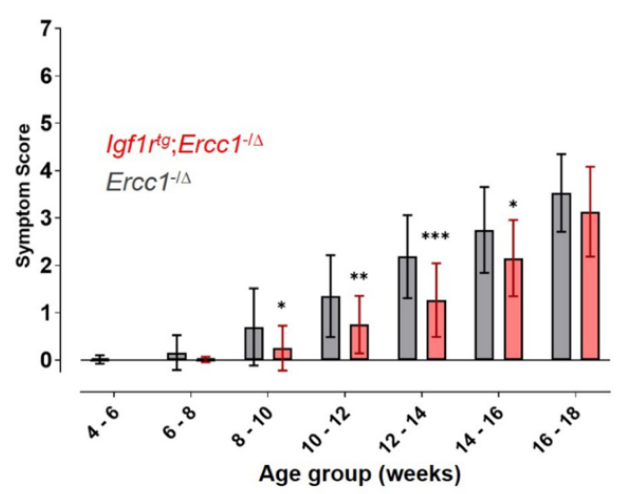

B

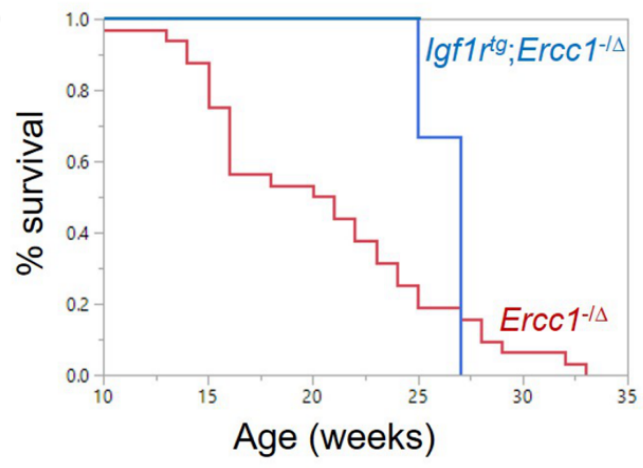

Figure 4. Healthspan and median lifespan are extended in progeroid $\boldsymbol{E r c c 1}^{-/ \Delta}$ mice overexpressing kinase-dead IGF1R in skeletal muscle. A. The onset and severity of kyphosis, ataxia, impaired ambulation, dystonia, tremors and poor body condition were measured twice per week as described in methods

\section{Conclusions}

We have generated a novel IGF1R $\mathrm{R}^{\mathrm{h} 407 \mathrm{H}}$ mouse line that carries a longevity-related variant enriched in human centenarians. This mouse line serves as a prototype for studying genetic-related longevity in humans and furthers the field of longevity research to promote healthy aging. Lifespan studies now are needed to confirm the longevity of IGF1 $\mathrm{R}^{\mathrm{hR} 407 \mathrm{H}}$ mice and to help show that the variant plays a role in human longevity. The mouse line serves as a platform for studying longevity associated genetic variants with high translational value for developing drugs that can target these variants to promote healthy aging. Finally, the customized CRISPR editing approach represents a prototype for generating additional longevity associated gene variant mouse lines to study relevance in aging and longevity.

\section{Declarations}

This work was supported by NIH U19 AG017221; The Nathan Shock Center of Excellence for the basic Biology of Aging (P30AG038072) (N. B.); Einstein-Paul Glenn Foundation for Medical Research Center for the Biology of Human Aging (N. B.); NIH R37 AG18381 (Barzilai Merit Award).

\section{Acknowledgements}

We thank Ruby Mangalindan, Robert Hunter and the Transgenic Resources Program (UW, Department of Comparative Medicine) for help generating the IGF1R $\mathrm{R}^{\mathrm{hR} 407 \mathrm{H}}$ transgenic mice.

\section{Competing interests}

The authors declare there are no competing interests.

\section{References}

1. Kenyon CJ (2010) The genetics of ageing. Nature 464: 504-512.

2. Hakuno F, Takahashi SI (2018) 40 years of IGF1: IGF1 receptor signaling pathways. $J$ Mol Endocrinol 61: T69-T86.

3. Suh Y, Atzmon G, Cho MO, Hwang D, Liu B, et al. (2008) Functionally significant insulin-like growth factor I receptor mutations in centenarians. Proc Natl Acad Sci USA 105: 3438-3442.

4. Tazearslan C, Huang J, Barzilai N, Suh Y (2011) Impaired IGF1R signaling in cells expressing longevity-associated human IGF1R alleles. Aging Cell 10: 551-554.

5. Fernández AM, Kim JK, Yakar S, Dupont J, Hernandez-Sanchez C, et al. (2001) Functional inactivation of the IGF-I and insulin receptors in skeletal muscle causes type 2 diabetes. Genes Dev 15: 1926-1934.

6. Doench JG, Fusi N, Sullender M, Hegde M, Vaimberg EW, et al. (2016) Optimized sgRNA design to maximize activity and minimize off-target effects of CRISPR-Cas9. Nat Biotechnol 34: 184-191.

7. Sanson KR, Hanna RE, Hegde M, Donovan KF, Strand C, et al. (2018) Optimized libraries for CRISPR-Cas9 genetic screens with multiple modalities. Nat Commun 9: $1-15$.

8. Kim HK, Min S, Song M, Jung S, Choi JW, et al. (2018) Deep learning improves prediction of CRISPR-Cpf1 guide RNA activity. Nat Biotechnol 36: 239-241.

9. Chen S, Lee B, Lee AY-F, Modzelewski AJ, He L (2016) Highly Efficient Mouse Genome Editing by CRISPR Ribonucleoprotein Electroporation of Zygotes. J Biol Chem 291: 14457-14467.

10. Mehravar M, Shirazi A, Nazari M, Banan M (2019) Mosaicism in CRISPR/Cas9mediated genome editing. Dev Biol 445: 156-162.

11. Gregg SQ, Robinson AR, Niedernhofer LJ (2011) Physiological consequences of defects in ERCC1-XPF DNA repair endonuclease. DNA Repair 10: 781-191. 